Article

\title{
An Optofluidic Lens Array Microchip for High Resolution Stereo Microscopy
}

\author{
Mayurachat Ning Gulari ${ }^{1}$, Anurag Tripathi ${ }^{2}$, Mostafa Ghannad-Rezaie ${ }^{3}$ and Nikos Chronis ${ }^{1,2,3, *}$ \\ 1 Macromolecular Science and Engineering, University of Michigan, Ann Arbor, MI 48109, USA; \\ E-Mail: toning@umich.edu \\ 2 Department of Mechanical Engineering, University of Michigan, Ann Arbor, MI 48109, USA; \\ E-Mail: anuragt@umich.edu \\ 3 Department of Biomedical Engineering, University of Michigan, Ann Arbor, MI 48109, USA; \\ E-Mail:mostafa@umich.edu
}

* Author to whom correspondence should be addressed; E-Mail: chronis@umich.edu;

Tel.: +1-734-763-0154; Fax: +1-734-615-6647.

Received: 9 June 2014; in revised form: 10 August 2014 / Accepted: 20 August 2014 /

Published: 28 August 2014

\begin{abstract}
We report the development of an add-on, chip-based, optical module-termed the Microfluidic-based Oil-immersion Lenses ( $\mu \mathrm{OIL}$ ) chip - which transforms any stereo microscope into a high-resolution, large field of view imaging platform. The $\mu$ OIL chip consists of an array of ball mini-lenses that are assembled onto a microfluidic silicon chip. The mini-lenses are made out of high refractive index material (sapphire) and they are half immersed in oil. Those two key features enable submicron resolution and a maximum numerical aperture of $\sim 1.2$. The $\mu \mathrm{OIL}$ chip is reusable and easy to operate as it can be placed directly on top of any biological sample. It improves the resolution of a stereo microscope by an order of magnitude without compromising the field of view; therefore, we believe it could become a versatile tool for use in various research studies and clinical applications.
\end{abstract}

Keywords: ball lens; microscope; oil immersion lens; microlens; stereo microscope; high resolution imaging; add-on module; optofluidics; microfabrication 


\section{Introduction}

Stereo microscopes are low-end, low-cost, imaging instruments, mainly used in the field of biology and medicine [1]. They provide 3D images; have long working distances, large field of view and depth of focus. They are also modular, as the manufacturers provide a wide variety objectives, stands and illumination accessories. Because of these advantages, they are commonly used in the lab or in the hospital for bacteria counting, organism and tissue imaging, as well as for dissecting and inspecting specimens [2-5]. Stereo microscopes are also used in geology and archaeology as well as for inspecting microparts and electronics, making jewelry and repairing watches [6].

Despite the advantages mentioned above, stereo microscopes have limited resolution (typically $10-20 \mu \mathrm{m})$ and magnification $(1 \times-6 \times$ without considering the magnification from the eyepiece) [6] when compared to the submicron resolution and magnification of up to $100 \times$ of expensive compound microscopes. In particular, low resolution is a critical shortcoming in low-end imaging systems as it prevents their use in clinical applications where cellular and sub-cellular imaging is needed.

Research efforts towards low cost, compact imaging systems have focused on utilizing microlenses [7-10] and microlens arrays [11-26] optofluidic microscope [27,28] or even tomographic imaging methods $[29,30]$. Although these approaches demonstrated high-resolution imaging, they typically lack the flexibility and versatility of a stereo microscope where the user can easily adjust the focus and magnification or the illumination conditions depending on the sample that is being imaged. An add-on optical module, e.g., a lens attachment for high-resolution imaging [21,31-34], would be an attractive solution in order to maintain the intended generic use of a stereo-microscope.

Here, we report the development of a compact and inexpensive optical, add-on module that transforms any stereo microscope into a high resolution imaging system without compromising the field of view. The add-on module - a Microfluidic-based Oil-immersion Lens ( $\mu$ OIL) chip — consists of an array of ball mini-lenses of excellent optical quality, which are microfluidically connected and half immersed in oil. The use of the $\mu$ OIL chip is simple as it can be mounted on top of any biological sample and imaged through the stereo microscope by simply adjusting the focus. The $\mu$ OIL chip is microfabricated on a wafer-level process, while the ball lenses are commercially available. The combined stereo microscope/ $\mu$ OIL chip system is an ideal tool for research studies and clinical applications where sub-micron and large field of view imaging is required.

\section{The $\mu$ OIL Chip}

\subsection{Architecture of the $\mu$ OIL Chip}

The $\mu$ OIL chip consists of a $4 \times 4$ array of $1 \mathrm{~mm}$ in diameter ball mini-lenses. They are precisely seated on top of an array of $970 \mu \mathrm{m}$ diameter microwells, etched on a silicon chip (Figure 1a,b). We use the term "ball mini-lenses" to indicate that they are small in size but they are not microfabricated. Those commercially available, ball mini-lenses are made of a high refractive index material (sapphire, refractive index 1.77, Edmund Optics, Barrington, NJ, USA, catalogue number 43-638) that greatly increases the numerical aperture (NA) of the system. They are half immersed in microscope oil, and half exposed to air. As a result, they act as doublet lenses, resembling the front end of a high-end, oil immersion microscope objective. We choose those mini-lenses as they have optical quality surface 
finish, tight tolerances (diameter, sphericity) and excellent broadband transmission. The silicon microwells are connected to each other through an open-air microfluidic network that distributes the oil uniformly to the entire array. The silicon chip is bonded to a thin glass substrate in order to seal the bottom of the microwells. The dimensions of the various elements of the $\mu$ OIL chip were chosen, such that the focal plane of the ball mini-lenses is located at the bottom surface of the glass chip.

The sample, which typically sits on a transparent substrate (e.g., a microscope glass slide), is placed underneath the $\mu$ OIL chip at a fixed distance from the ball mini-lens array. That distance is accurately defined by the thickness of a spacer. The spacer can be a thin photoresist film that is patterned on the bottom of the glass substrate or stacked glass chips (typically 100-300 $\mu \mathrm{m}$ thick) placed underneath each side of the $\mu$ OIL chip. The latter option is recommended when a large field of view (FOV) is desired and high resolution is not that critical. When the sample/ $\mu$ OIL chip assembly is imaged through a stereoscope (Figure 1c), a high resolution and magnified image of the sample is obtained. Using an array of 16 ball lenses fully assembled on the $\mu$ OIL chip (Figure 1b), one can observe simultaneously multiple areas of interest.

Figure 1. (a) Cross section schematic of the $\mu \mathrm{OIL}$ chip; (b) A picture (left) of the microfabricated $\mu \mathrm{OIL}$ chip (scale bar: $3 \mathrm{~mm}$ ) and a close up view (right) of the microwell array with no ball mini-lenses (scale bar, $1 \mathrm{~mm}$ ); and (c) The combined $\mu$ OIL chip/stereo microscope system. The $\mu$ OIL chip is placed directly on top of the sample of interest.

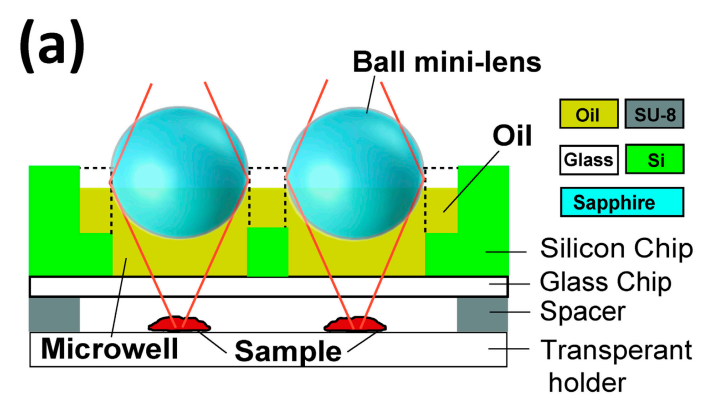

(c)

(b)
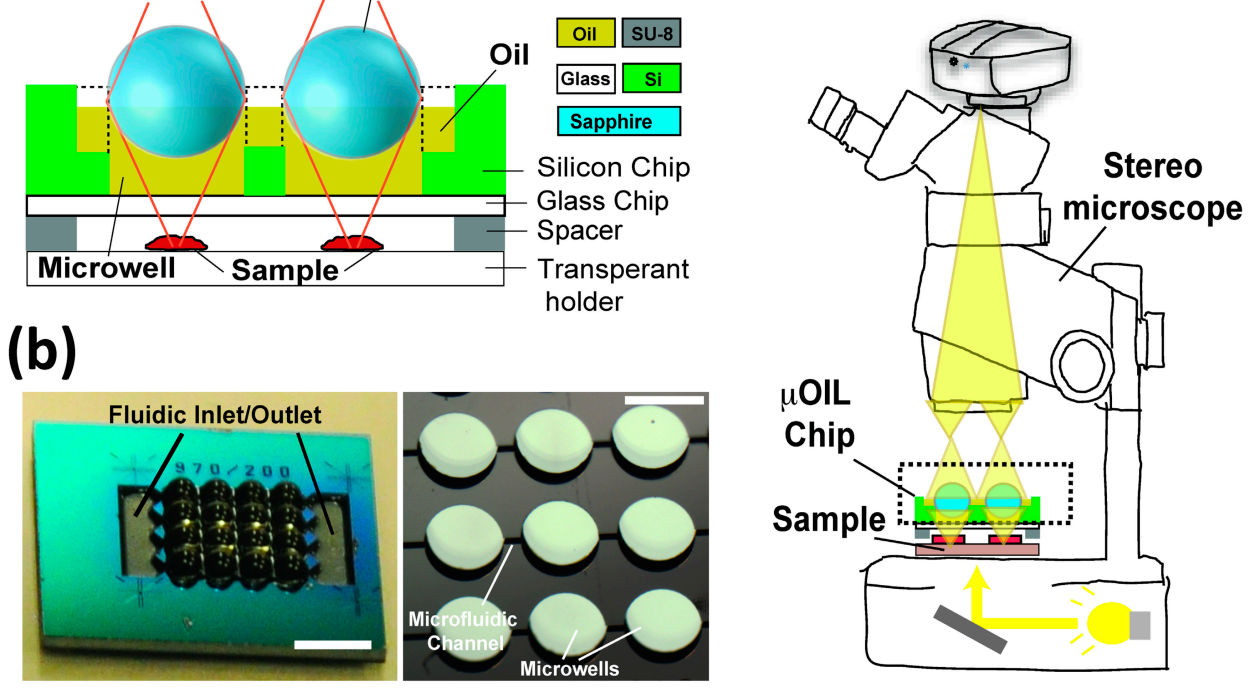

\subsection{Microfabrication}

The $\mu$ OIL chip is fabricated using a 2-mask, standard silicon micromachining processes. The fabrication process consists of the following steps (Figure 2a): (1) A $400 \mu \mathrm{m}$ thick silicon wafer is thermally oxidized to form a $200 \mathrm{~nm}$ thick $\mathrm{SiO}_{2}$ film. The $\mathrm{SiO}_{2}$ film is photolithographically patterned and Hydrofluoric acid-etched to create a hard masking layer for etching the microfluidic network; (2) A $5 \mu \mathrm{m}$ thick photoresist film is then patterned using the second mask that contains the microwell array and a deep reactive ion etching (DRIE) step is performed to partially etch $200 \mu \mathrm{m}$ out of the $400 \mu \mathrm{m}$ microwell total thickness; (3) The photoresist is then stripped to expose the $\mathrm{SiO}_{2}$ film. A 
wafer-through DRIE step is then performed to obtain the $200 \mu \mathrm{m}$ thick microfluidic network and the microwells; (4) The silicon wafer is anodically bonded to a $100 \mu \mathrm{m}$ thick Pyrex wafer and the bonded wafer is diced. A $5 \mu \mathrm{m}$ thick SU-8 film - the spacer is patterned at the bottom of each individual chip. Instead of using the SU-8 film, multiple glass coverslips can be stacked on top of each other and glued on the sides of the chip if it is desired to increase the distance between the $\mu$ OIL chip and the sample; (5) The microfluidic network is filled with microscope immersion oil (Cargillis labs \#159, Cedar Grove, NJ, USA, refractive index 1.52 and (6) the ball mini-lenses are manually assembled on the top of the oil-filled microwell array and kept in place by surface tension. They are separated from the bottom surface of the microwell array by $\sim 20 \mu \mathrm{m}$. Before use, the $\mu$ OIL module is placed on a flat surface for $30 \mathrm{~min}$ to let oil evenly distribute into the microwell array. The assembled $\mu$ OIL module is then ready for use: It can then be placed on top of any specimen to obtain high resolution images through a stereoscope. The $\mu \mathrm{OIL}$ module can be re-used indefinitely as long as it is kept in a dry environment (to minimize oil evaporation). It can also be cleaned and re-assembled if needed. For long term use, a thin parylene film can also be deposited to completely seal the $\mu$ OIL module.

Figure 2. (a) Microfabrication process and (b) Critical dimensions of the $\mu$ OIL chip. The focal length measured from the center of the ball mini-lens is $\sim 620 \mu \mathrm{m}$. The dotted lines represent the microwell sidewalls that are not visible in the highlighted cross section.

(a)

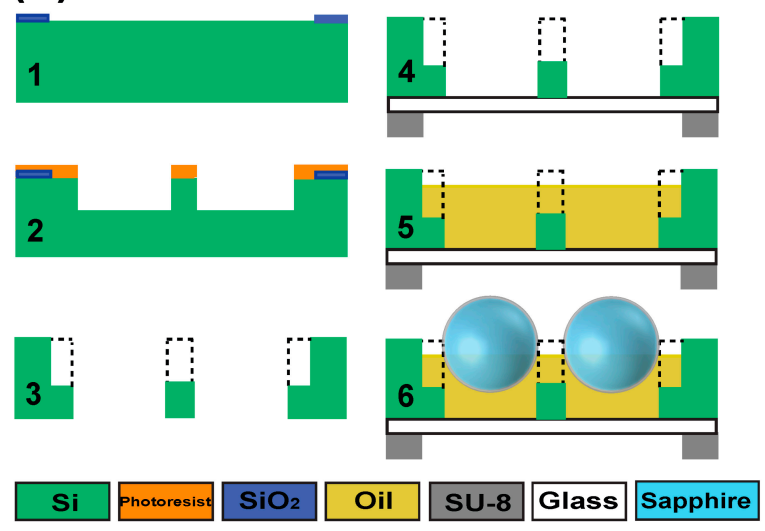

(b)

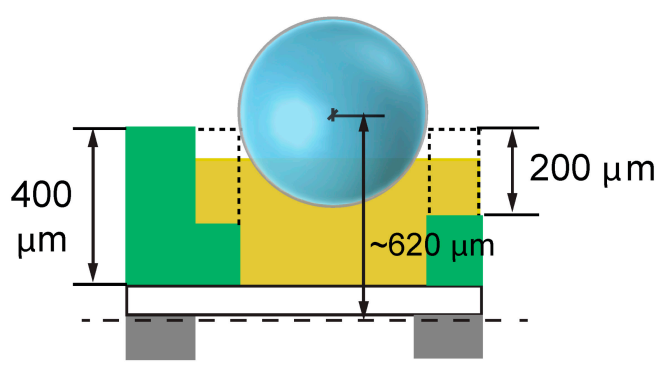

\section{Results and Discussion}

\subsection{The $\mu$ OIL Module}

\subsubsection{Material Considerations}

The amount of light collected by a lens that also affects its resolution can be quantified by its numerical aperture (NA):

$$
\mathrm{NA}=n_{\mathrm{M}} \cdot \sin \theta
$$

where $n_{\mathrm{M}}$ is the refractive index of the medium that the lens is immersed into and $\theta$ is the half angle of the cone of rays collected by the lens. The maximum half angle $\theta$ and therefore the maximum NA can be obtained when the sample is located at the focal length of the lens. In order to increase the NA, one should increase the refractive index of the medium and/or increase the angle $\theta$. The former task can be 
achieved by using media that have high refractive index (e.g., oil or water). The latter one can be achieved by combining the high refractive index media with a high refractive index lens.

Our $\mu$ OIL chip has similar behavior, which can be described by Equation (1): The effect of the refractive index of the medium and the ball lens material are critical for achieving high NA. We choose to use microscopy oil as a medium in order to match the refractive index of the glass chip that seals the bottom of the microwell array. The use of oil reduces the total internal reflection of optical rays at the glass/oil interface, resulting in an increase in angle $\theta$ (the focal length also increases in this case but the effect compared to the increase in angle $\theta$ is less significant) (Figure 3a). Microscopy oil also does not fluoresce and does not evaporate as fast as other media. In addition, by using a high refractive index lens material (e.g., sapphire, refractive index $n_{\mathrm{L}}=1.77$ ), the focal length decreases and the optical cone widens (angle $\theta$ increases) (Figure $3 b$ ).

Figure 3. The effect of the refractive index of the immersion medium (a) and ball lens (b) on the ray collection angle $\theta_{\mathrm{o}}$ Values for angle $\theta_{\mathrm{o}}$ are theoretically estimated by thick-lens equations (see supplementary materials and Figure S1).

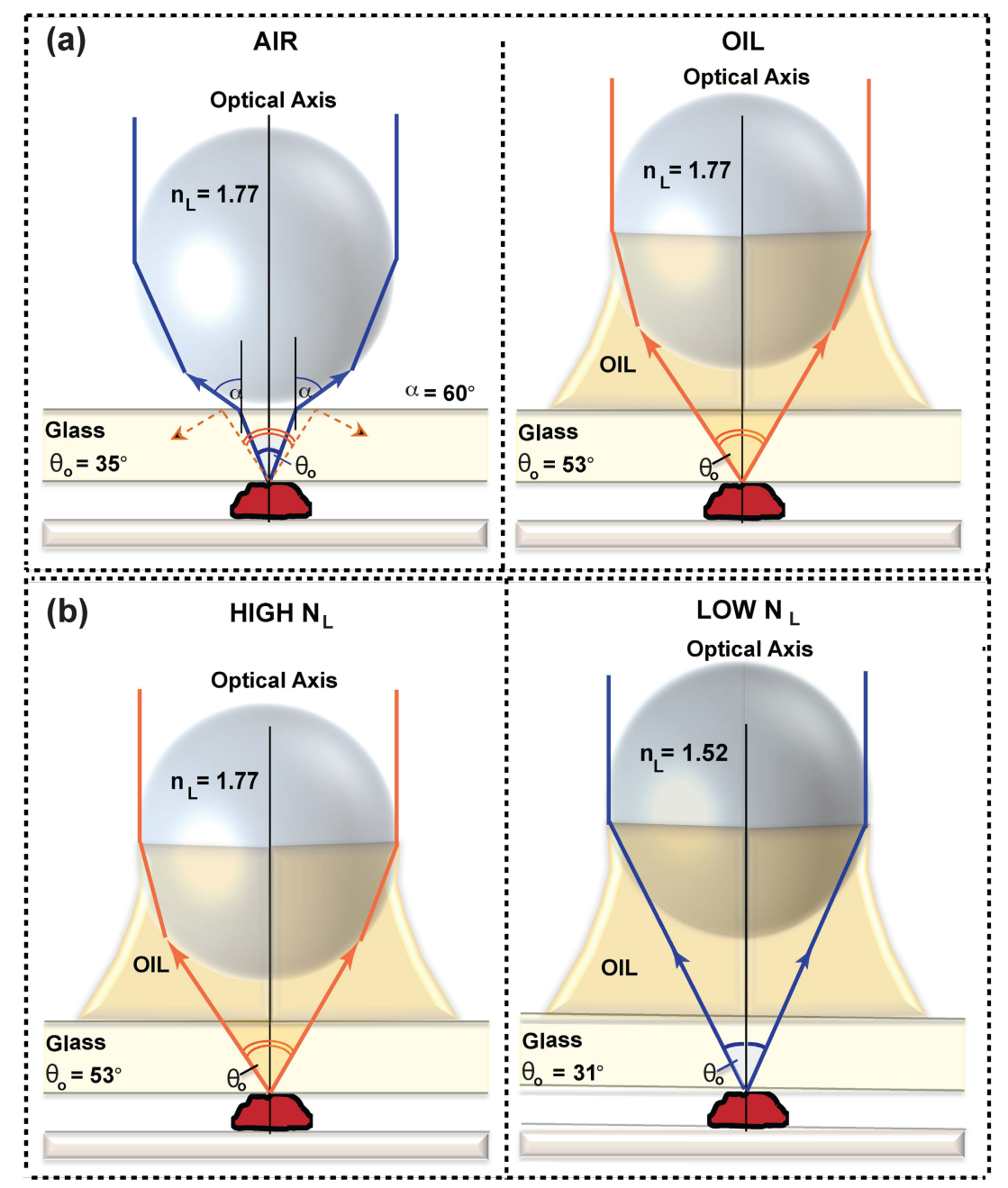

This specific combination of materials results in a high optical performance of the $\mu$ OIL module (Table 1) which is excellent for high-resolution imaging (see supplementary materials for calculating the various optical properties of the $\mu$ OIL module). We should emphasize that due to the modular 
assembly of the ball mini-lenses, the oil and the silicon/glass chip, the end user has the ability to custom-make his/her $\mu$ OIL module.

Table 1. Properties of $\mu$ OIL chip.

\begin{tabular}{cc}
\hline Parameter & Value \\
\hline Ball mini-lens Diameter $(D)$ & $D=1 \mathrm{~mm}$ \\
Ball mini-lens Refractive index $\left(n_{\mathrm{L}}\right)$ & $n_{\mathrm{L}}=1.77$ (sapphire) \\
Back Focal Length (BFL) & $124 \mu \mathrm{m}$ (see supplementary materials) \\
Numerical Aperture (NA) & 1.2 (from Equation (2)) \\
Lens power $(\varphi)$ & $1.6 \mathrm{~mm}^{-1}$ (from Equation $\left.(3)\right)$ \\
Magnification & $\sim 130 \times($ see Figure 5) \\
Resolution (center) & $0.7 \mu \mathrm{m}$ (see Figure 7) \\
Depth of Field (DOF) & $0.38 \mu \mathrm{m}$ (from $\left.\lambda / \mathrm{NA}^{2}\right)[13,19]$ \\
Field of View (FOV) & $60-140 \mu \mathrm{m} /$ lens (see Figure 8$)$ \\
$F$-number & 0.62 (from EFL $* / D$ ) \\
\hline
\end{tabular}

Notes: $*$ EFL: the effective focal length (see supplementary materials); $\lambda$ : the wavelength.

\subsubsection{The Numerical Aperture (NA) of the $\mu$ OIL Chip}

In order to estimate the NA of the $\mu$ OIL module, we modeled the ball lens as a thick lens [35] that is half immersed in medium and half immersed in air. We neglected any refraction of light that might take place between the focal plane and the medium. In this case, the NA can be expressed as a function of refractive index of the lens material $\left(n_{\mathrm{L}}\right)$, the refractive index of the medium $\left(n_{\mathrm{M}}\right)$, the lens power $(\varphi)$ and the lens radius (see supplementary materials):

$$
\begin{gathered}
\mathrm{NA}=n_{\mathrm{M}}\left(\frac{\varphi R n_{\mathrm{L}}}{\varphi R n_{\mathrm{L}}+n_{\mathrm{M}} n_{\mathrm{L}}-2\left(n_{\mathrm{L}}-1\right) n_{\mathrm{M}}}\right) \\
\varphi=\frac{1}{R}\left(n_{\mathrm{M}}+1-\frac{2 n_{\mathrm{M}}}{n_{\mathrm{L}}}\right)
\end{gathered}
$$

To better understand the dependence of NA on $n_{\mathrm{M}}$ and $n_{\mathrm{L}}$, we plotted the NA versus $n_{\mathrm{L}}$, when the ball mini-lens is immersed in four different media: air, water, oil and polydimethylsiloxane (Figure 4). Air, water and oil are widely used media in standard optical microscopy. To cover the low end of the $n_{\mathrm{M}}$ spectrum, we choose PDMS as an alternative low-cost solution. The $n_{\mathrm{L}}$ of the lens was varied from 1.4 to 2, as those values represent refractive indices of off-the-shelve ball lenses (e.g., N-BK7 $\left(n_{\mathrm{L}}=1.516\right)$, Ruby $\left(n_{\mathrm{L}}=1.77\right)$, S-LAH79 $\left(n_{\mathrm{L}}=2\right)$, etc. $)$. In all calculations, we did not take into account the aperture created by the silicon well (the silicon well is $30 \mu \mathrm{m}$ smaller in diameter than the ball lens diameter) and we assumed that the top half of the ball-lens is exposed to air. We want to note that in reality, the size of the holder aperture (i.e., silicon well) needs to be taken into account since light blocked by the smaller aperture will reduce the maximum cone angle of the rays thus effect NA value. For all practical purposes, we also assumed that the sample is located at the focal plane. The results quantitatively validate our expectations: the higher the $n_{\mathrm{M}}$ and $n_{\mathrm{L}}$ the higher the NA. For ball mini-lenses of small refractive index $\left(n_{\mathrm{L}}=1.5-1.6\right)$, an increase of the refractive index of the immersion medium does not result a significant increase in the NA. The effect of refractive index of the immersion medium on the NA is significant (up to $\sim 50 \%$ ) when ball mini-lenses with high 
refractive indices are used (e.g., $\left.n_{\mathrm{L}}>1.7\right)$. The combination of microscope immersion oil $\left(n_{\mathrm{M}}=1.516\right)$ and sapphire ball mini-lens $\left(n_{\mathrm{L}}=1.77\right)$ results in a numerical aperture of NA $=\sim 1.2$ which is comparable to high-end oil or water immersion microscope objectives. For the result we report in this paper, the effective aperture is $320 \mu \mathrm{m}$ in diameter allowing a light collection cone angle of $53^{\circ}$ based on the geometry of Figure $3 b$.

Figure 4. Numerical Aperture (NA) of the $\mu \mathrm{OIL}$ chip versus refractive index of the ball mini-lens $\left(n_{\mathrm{L}}\right)$ for four different cases of immersion media as theoretically predicted by Equation (2).

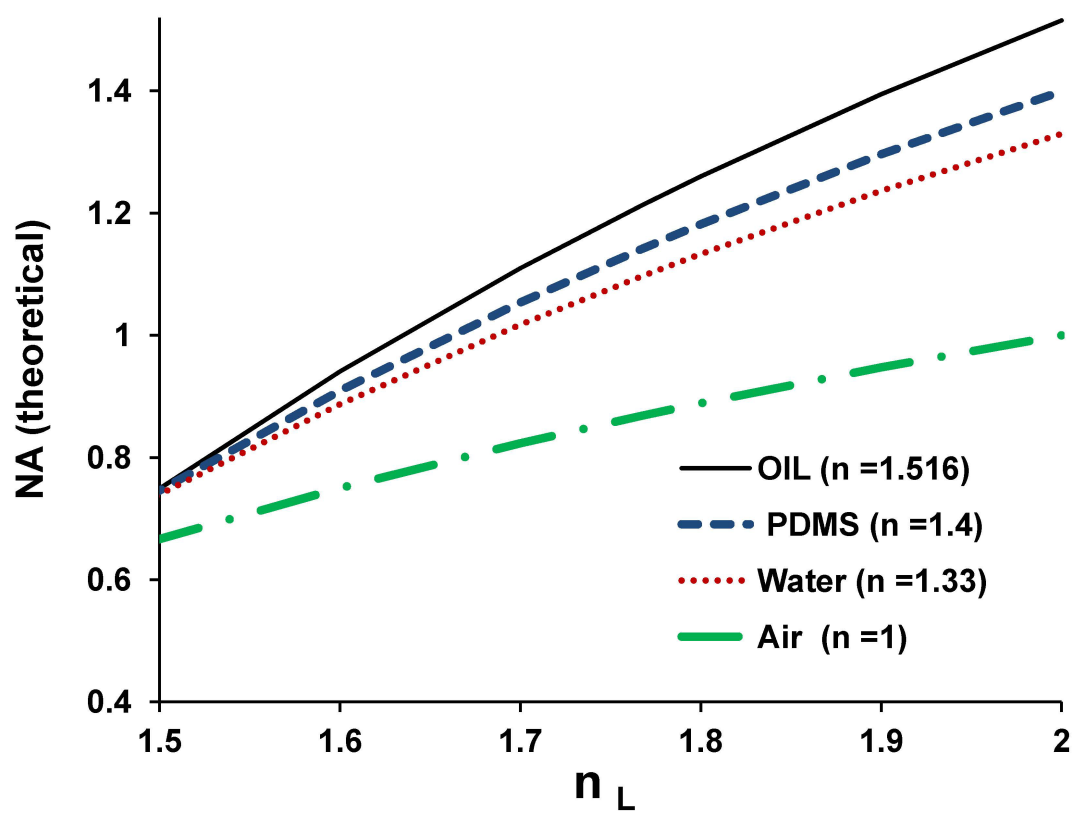

\subsubsection{Magnification and NA versus Spacer Thickness}

We performed optical simulations using Optics Software for Layout and Optimization (OSLO) software to obtain the magnification and numerical aperture as a function of the distance of the sample from the focal plane of the $\mu \mathrm{OIL}$ chip (the focal plane is located at the bottom glass surface of the $\mu$ OIL chip). This distance coincides with the thickness of the spacer. For all practical purposes, we assumed a minimum spacer thickness of $5 \mu \mathrm{m}$ (that is the thickness of the SU-8 film) and that the medium between the sample and the bottom glass surface of the $\mu$ OIL chip is water. In this case, the numerical aperture and magnification are $\sim 1 \times$ and $\sim 130 \times$ respectively. As expected, when the spacer thickness increases, both the magnification and NA decrease. Spacer thickness has a major impact on the magnification, as the magnification decay has an exponential trend. At a spacer thickness of $900 \mu \mathrm{m}$ the magnification and the NA is $1 \times$ and $\sim 0.01$ respectively (data not shown), beyond that point the sample is being demagnified. We want to emphasize that this is the magnification obtained from the $\mu$ OIL chip alone. For the combined stereo microscope/ $\mu$ OIL chip system, the total magnification is calculated by multiplying the magnification of the $\mu \mathrm{OIL}$ chip and the magnification from the stereo microscope. 
Figure 5. Dependence of the magnification and NA of the $\mu$ OIL chip on spacer thickness.

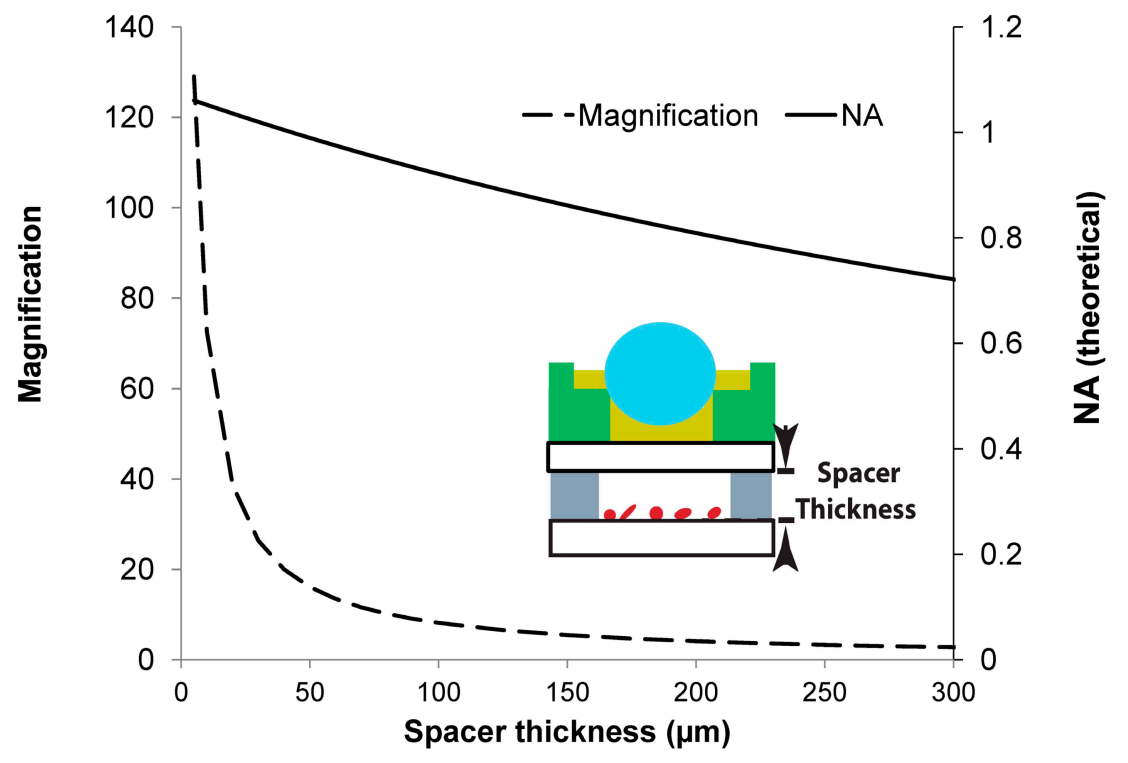

\subsection{The Combined $\mu$ OIL Chip/Stereo Microscope Assembly}

We evaluated the optical performance of a standard stereo microscope (Olympus SZ61, Tokyo, Japan) when the $\mu$ OIL chip is used as an add-on module. We placed the $\mu$ OIL module on top of various samples (test charts, blood cells) and analyzed the obtained images. The stereo microscope we used had a magnification range from $0.67 \times$ to $4.5 \times$ and was equipped with a 3.3 megapixel CCD (MicroPublisher 3.3RTV, with $0.5 \times$ de-magnifying lens in from of the camera, British Columbia, Canada). In all tests described below, we set the magnification of the stereo microscope to its maximum value of $4.5 \times$ that corresponds to a maximum NA of $\sim 0.07$ and a maximum resolution of $\sim 10 \mu \mathrm{m}$ [36]. We used bright field illumination in transmission mode by adjusting the mirror at the base of the stereoscope at $\sim 45^{\circ}$. The use of the $\mu$ OIL chip as a module greatly enhances the resolution of the stand-alone stereo microscope (Figure 6). Using the stereo microscope alone, the finest pattern from a standard resolution plate (U.S. Air Force-USAF1951) is blurred and none of the lines can be resolved (Figure 6a). When the $\mu$ OIL chip is placed on top of the resolution plate, the narrowest lines $(0.775 \mu \mathrm{m}$ in width $)$ are resolved (Figure 6b). Although, there is noticeable aberration away from the center of field of view.

\subsubsection{Resolution}

To obtain the optical resolution of the combined system, we measured the image contrast of a resolution plate (USAF1951) that has equally spaced line patterns of decreasing width. The dependence of the image contrast on the line width represents the modulation transfer function of the combined system, which is a widely accepted method for obtaining the resolution of an optical system [37]. The acquired images were taken within $30 \mu \mathrm{m}$ of the center of the field of view and analyzed using Metamorph software and the percentage of the image contrast averaged over all color channels versus the number of line pairs per millimeter was obtained (Figure 7). In addition, we tested the effect on the resolution of a thin $(\sim 125 \mathrm{~nm})$ parylene film that was deposited on top of the $\mu$ OIL chip. As discussed above, the parylene film can permanently seal the $\mu$ OIL chip in order to prevent evaporation of the oil as well as to serve as an anti-reflective coating [38]. 
Figure 6. Images of a resolution plate (USAF1951) obtained from a stereo microscope alone (a) and from the combined $\mu$ OIL chip/stereo microscope system (b). Scale bar: $10 \mu \mathrm{m}$ in (a) and (b).
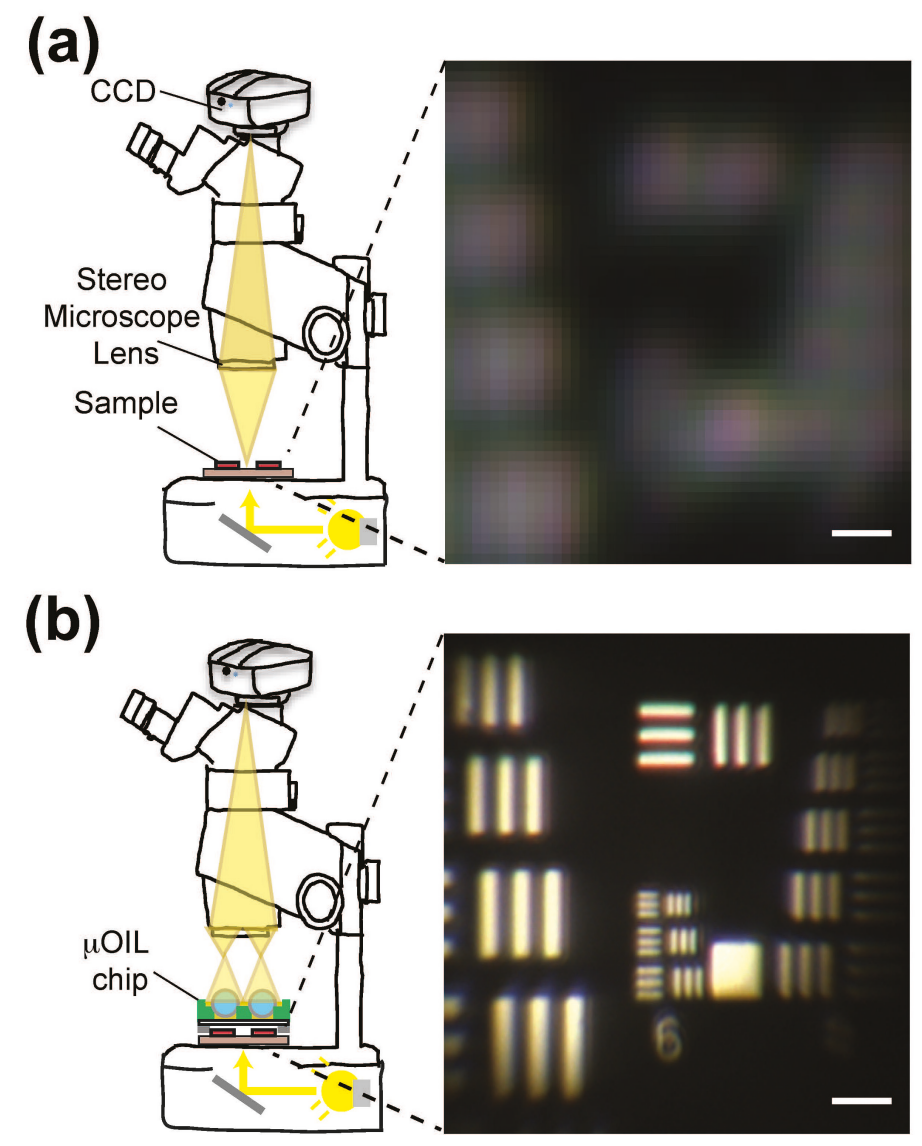

Figure 7. Image contrast (\%) versus line pairs per $\mathrm{mm}$ for the combined system. The sample was a resolution plate that was imaged in air. The spacer thickness was $5 \mu \mathrm{m}$ and NA (theoretical) is $\sim 0.79$.

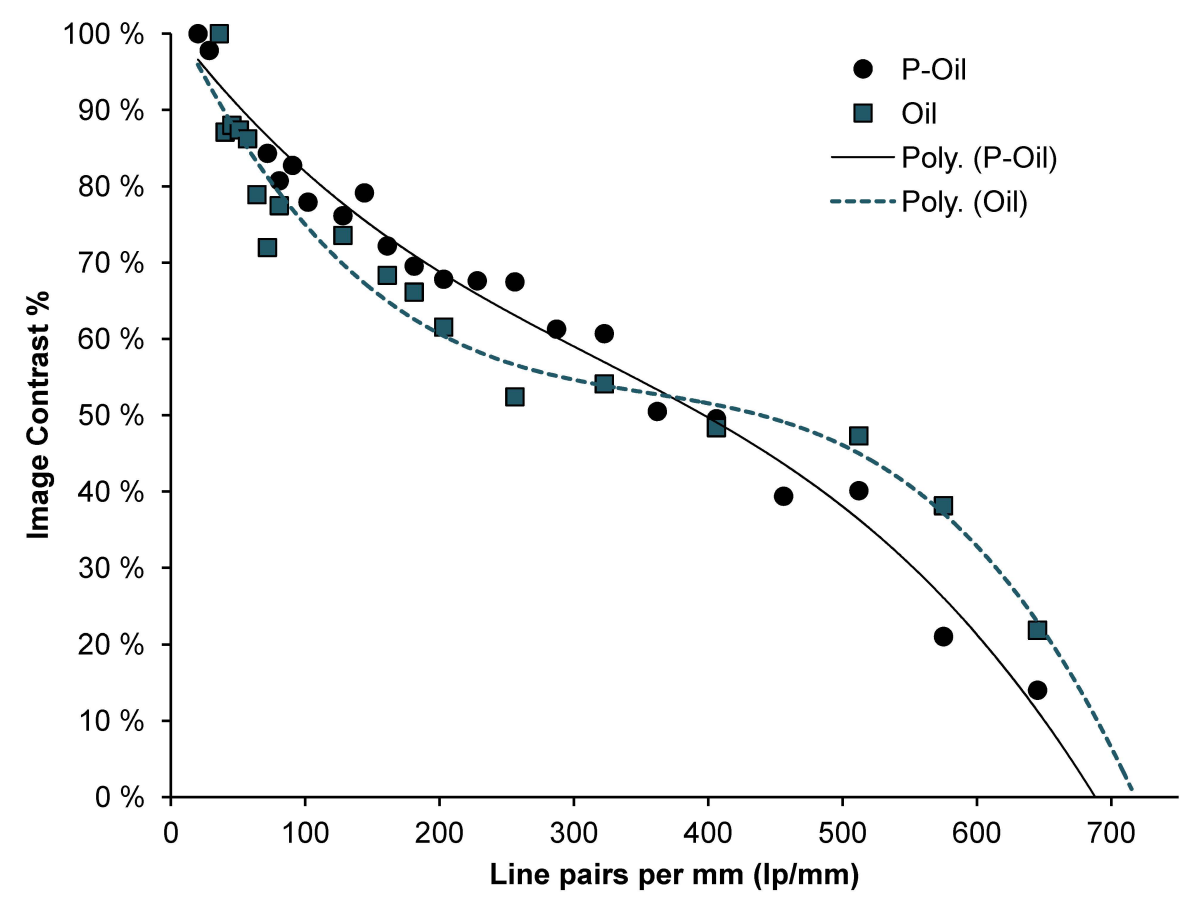


The experimental results indicate that the image contrast percentage decreases continuously as the number of line pairs per $\mathrm{mm}$ increases. All the lines from the resolution plate even the smallest lines with a width of $0.775 \mu \mathrm{m}$ (corresponding to 645 line pairs $/ \mathrm{mm}$ ) can be imaged clearly using both the non-coated $\mu$ OIL chip and the parylene-coated $\mu$ OIL chip. However, at 400 line pairs $/ \mathrm{mm}$ and above, the parylene-coated $\mu$ OIL chip resulted in a slight degradation in the optical performance as compared to the non-coated $\mu$ OIL chip. As the minimum feature size of the resolution plate is larger than the anticipated maximum resolution, we can only estimate the latter one by extrapolating the modulation transfer function until the image contract becomes zero. Using this approach, the theoretical resolution for the non-coated and the parylene-coated $\mu \mathrm{OIL}$ chip is $715 \mathrm{lp} / \mathrm{mm}(0.7 \mu \mathrm{m}$ line width) and $690 \mathrm{lp} / \mathrm{mm}(0.73 \mu \mathrm{m}$ line width) respectively. We should also emphasize that the obtained results are not limited by the camera resolution which was measured to be $0.15 \mu \mathrm{m} /$ pixel at maximum magnification.

\subsubsection{Field of View (FOV)}

We experimentally obtained the change in the FOV of the combined system versus spacer thickness (Figure 8). The FOV was measured by imaging a commercially available grid distortion target (Thorlabs, catalog number R1L3S3P, Newton, NJ, USA,) that consists of $10 \mu \mathrm{m}$ wide metal squares. For each spacer thickness, we obtained the one dimensional intensity profile across the grid pattern (see supplementary Figure S2). The medium between the sample and the bottom glass surface of the $\mu$ OIL chip is water. The intensity was uniform at the center of the ball mini-lens where minimum image distortion is observed. The FOV was defined as the diameter of an area where the intensity is above $80 \%$ of the maximum intensity. Although such a criterion is subjective, we experimentally observed that the $80 \%$ cut-off threshold was a rather conservative value for most practical application (e.g., for cell counting). For high-resolution imaging, the spacer thickness needs to be minimized (e.g., $5 \mu \mathrm{m}$ ), which results in a minimum FOV in the range of $\sim 60 \mu \mathrm{m} /$ lens. Increasing the spacer thickness resulted in a linear increase in the FOV at the expense of resolution.

Figure 8. (a) Field of view (FOV) and NA versus spacer thickness. The theoretical NA values were calculated from thick-lens equation (see supplementary). (b) Image of $10 \mu \mathrm{m}$ grid using a $5 \mu \mathrm{m}$ spacer (I) and a 250 thick $\mu \mathrm{m}$ spacer (II). Scale bar: $20 \mu \mathrm{m}$ in (b).

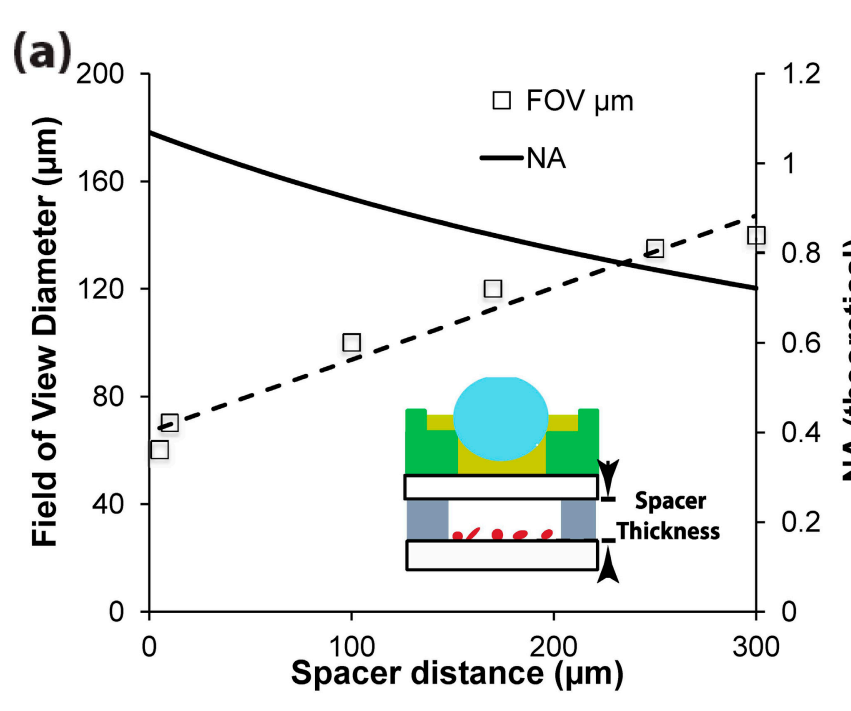

(b)

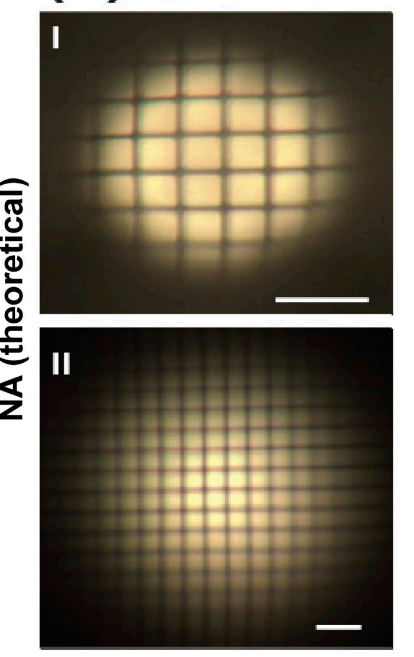




\subsubsection{Biological Imaging}

In order to demonstrate the practical usefulness of the $\mu$ OIL module, we imaged various biological samples using the combined system. In particular, we imaged: a whole, undiluted blood smear obtained from a patient diagnosed with sickle-cell disease (Figure 9a,b). Cells were stained with Wright and Giemsa stain and imaged using transmission brightfield illumination (as illustrated in Figure 9). White, normal red and sickle red blood cells and platelets were clearly visible and distinguishable. If the $\mu \mathrm{OIL}$ module is removed, then the stereomicroscope alone cannot resolve single cells (Figure 9c,d).

Figure 9. Wright and Giemsa stained blood smear from a patient with sickle-cell disease: neutrophils (a) and sickle cells (b) are clearly visible. In (c, d), the same blood smear sample as imaged from the stereo microscope alone. Scale bars: $10 \mu \mathrm{m}$ in (a), (b), (d); $50 \mu \mathrm{m}$ in (c).

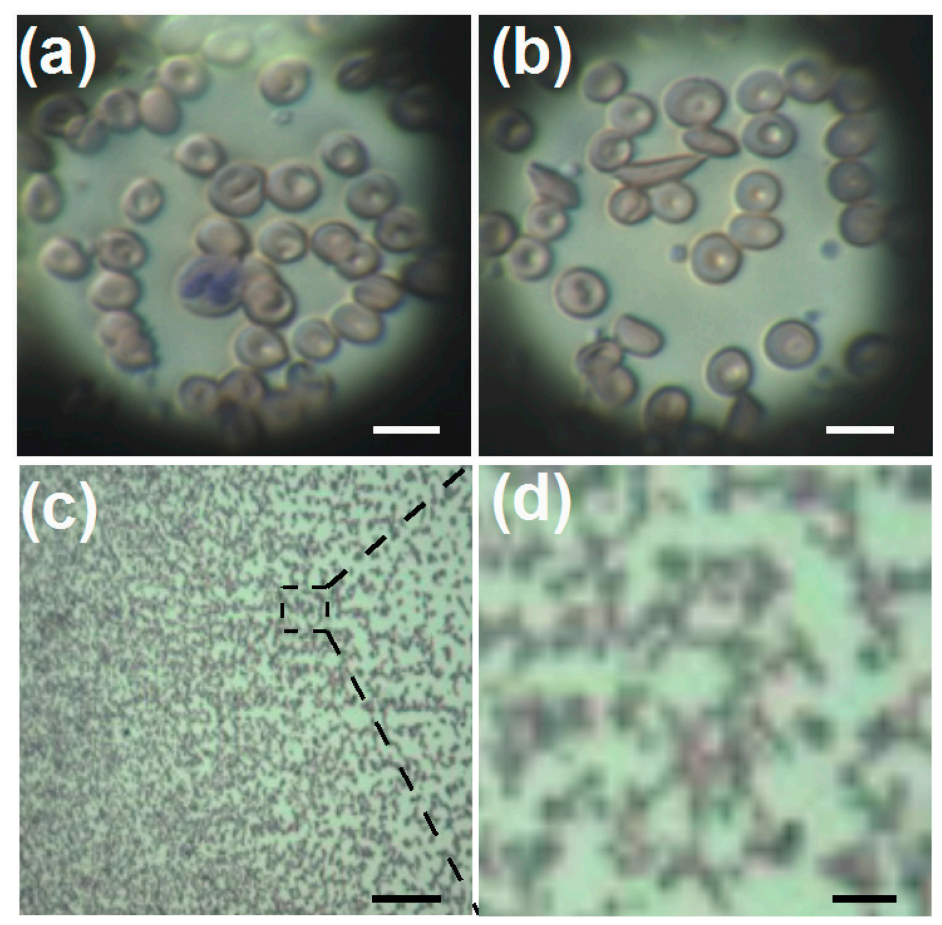

In all the above experiments, the magnification of the stereo microscope was $4.5 \times$. In this magnification, the combined system with the 1 inch CCD detector can simultaneously obtain images from 4 to 6 mini-lenses. To increase the FOV and image the entire $\mu$ OIL chip (all 16 mini-lenses), one has to decrease the magnification of the stereo microscope to $\sim 2 \times$. Even in this case the FOV is $\sim 60 \mu \mathrm{m} /$ lens the shape of single cells from a smear test (Figure 10) is still visible. In order to obtain a sharp image from all mini-lenses in the array, minor focus adjustments using the stereomicroscope focus knob (within the array, it is possible to have variation up to $50 \mu \mathrm{m}$ ) might be required as imperfections in the microfabrication and mini-lens assembly process result in a lens-to-lens variation in the focal length within the array. Our future work will incorporate a better design of the $\mu$ OIL chip, better control of the fabrication process and tighter quality control assembly to improve the focal plan uniformity of the array. 
Figure 10. Left image: Imaging a blood smear using the entire $\mu$ OIL chip (from all 16 ball mini-lens). The magnification of the stereo microscope was set to $2 \times$. Right image is the magnified version of the image from the lens in the square box shown in the left image. Scale bar: $20 \mu \mathrm{m}$.
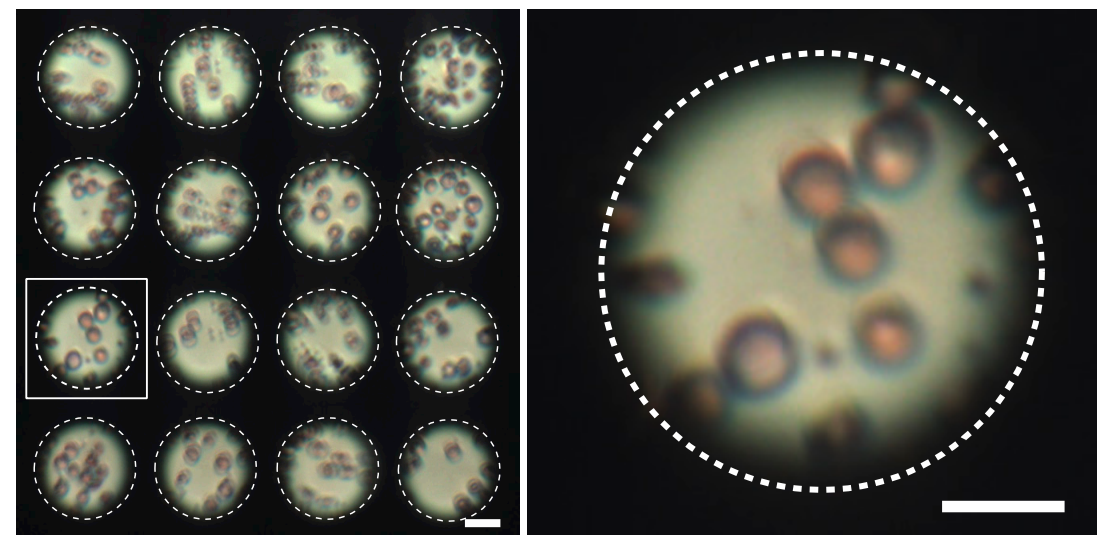

\section{Conclusions}

We have developed a simple-to-use, reusable and compact, microfabricated imaging module for high resolution, large field of view imaging applications. The key element of the design is the integration of a microfluidic chip with an array of 16 liquid-immersed, high refractive index ball mini-lenses. The integrated chip — termed the $\mu$ OIL — chip mimics the working principle of the front end of expensive, oil-immersion microscope objectives. The $\mu$ OIL chip alone has a maximum numerical aperture of 1.2 and a magnification of $130 \times$. It is attached directly to the sample of interest and then imaged under a standard micro stereoscope by simply adjusting the focus and/or the magnification of the stereo microscope.

We demonstrated that, when it is used as an add-on module to a stereo microscope, it can resolve submicron micron features (maximum resolution of $0.7 \mu \mathrm{m}$ ) from a variety of biological samples. We believe that the $\mu \mathrm{OIL}$ chip can bring high resolution, large field of view imaging capabilities to research and medical laboratories and clinics where a stereo microscope is the only available imaging tool.

\section{Supplementary Materials}

Supplementary materials can be accessed at: http://www.mdpi.com/2072-666X/5/3/607/s1.

\section{Acknowledgments}

This research made possible in part with support by the National Institutes of Health (NIH) Microfluidics in Biomedical Sciences Training Program (T32 EB005582-05) and the NIH Director's New Innovator Award (DP2OD006458). The authors would like to thank Onnop Srivannavit, Maria Daphne Bazopoulou, Amrita Ray Chaudhury, Alexandros Pantazis and Eleni Gourgou for useful discussions. We also want to thank Usha Kota and Christine Falkiewicz at the hematology lab, University of Michigan hospital for disease diagnostic training and providing sickle cell blood smear sample. All the devices were fabricated at the Lurie Nanofabrication Facility at the University of Michigan. 


\section{Author Contributions}

Mayurachat Ning Gulari performed the design, microfabrication, testing of the $\mu$ OIL chip, analyzed the data and wrote the manuscript. Anurag Tripathi and Mostafa Ghannad-Rezaie contributed on the design and microfabrication of the $\mu$ OIL chip. Nikos Chronis had oversight over the experiments and manuscript writing.

\section{Conflicts of Interest}

The authors declare no conflict of interest.

\section{References}

1. Bradbury, S.; Bracegirdle, D.B. Introduction to Light Microscopy; BIOS Scientific Publishers Limited: Milton Park, UK, 1998; p. 123.

2. Spellman, F.R. The Microscope. In Microbiology for Water and Wastewater Operators (Revised Reprint); CRC Press: Boca Raton, FL, USA, 1999; pp. 11-17.

3. Peyman, G.A.; Koziol, J.E.; Fried, W.I.; Sanders, D.R. Operating Microscope with Two Pairs of Stereo Eye-Piece Lenses. U.S. Patent 4,138,191, 6 February 1979.

4. Muller, O.; Biber, K.; Jakubowski, H.; Hanemann, G. Operation Microscope. U.S. Patent 4,448,498, 15 May 1984.

5. Fukaya, T.; Hamada, M.; Nakamura, S.; Hanzawa, T.; Kinukawa, M.; Ishikawa, T.; Fujiwara, H.; Tokunaga, S. Stereomicroscope. U.S. Patent 5,552,929, 3 September 1996.

6. Woolnough, L. Starting with Stereomicroscopes-Quekett Microscopical Club. Available online: http://www.quekett.org/starting/stereo-microscopes/stereo-microscopes (accessed on 8 May 2014).

7. Krupenkin, T.; Yang, S.; Mach, P. Tunable liquid microlens. Appl. Phys. Lett. 2003, 82, 316-318.

8. Cadarso, V.J.; Llobera, A.; Villanueva, G.; Dominguez, C.; Plaza, J.A. 3-D modulable PDMS-based microlens system. Opt. Express 2008, 16, 4918-4929.

9. Dong, L.; Agarwal, A.K.; Beebe, D.J.; Jiang, H. Adaptive liquid microlenses activated by stimuli-responsive hydrogels. Nature 2006, 442, 551-554.

10. Chen, J.; Wang, W.; Fang, J.; Varahramyan, K. Variable-focusing microlens with microfluidic chip. J. Micromech. Microeng. 2004, 14, 675-680.

11. Chronis, N.; Liu, G.; Jeong, K.H.; Lee, L. Tunable liquid-filled microlens array integrated with microfluidic network. Opt. Express 2003, 11, 2370-2378.

12. He, M.; Yuan, X.C.; Ngo, N.; Bu, J.; Tao, S. Low-cost and efficient coupling technique using reflowed sol-gel microlens. Opt. Express 2003, 11, 1621-1627.

13. Nussbaum, P.; Volkrl, R.; Herzig, H.P.; Eisner, M.; Haselbeck, S. Design, fabrication and testing of microlens arrays for sensors and microsystems. Pure Appl. Opt. 1996, 6, 617-636.

14. Aristizabal, S.L.; Cirino, G.A.; Montagnoli, A.N.; Sobrinho, A.A.; Rubert, J.B.; Hospital, M.; Mansano, R.D. Microlens array fabricated by a low-cost grayscale lithography maskless system. Opt. Eng. 2013, 52, doi:10.1117/1.OE.52.12.125101. 
15. Kozma, P.; Lehmann, A.; Wunderlich, K.; Michel, D.; Schumacher, S.; Ehrentreich-Förster, E.; Bier, F.F. A novel handheld fluorescent microarray reader for point-of-care diagnostic. Biosens. Bioelectron. 2013, 47, 415-420.

16. Orth, A.; Crozier, K. Microscopy with microlens arrays: High throughput, high resolution and light-field imaging. Opt. Express 2012, 20, 13522-13531.

17. Kim, J.Y.; Pfeiffer, K.; Voigt, A.; Gruetzner, G.; Brugger, J. Directly fabricated multi-scale microlens arrays on a hydrophobic flat surface by a simple ink-jet printing technique. J. Mater. Chem. 2012, 22, 3053-3058.

18. Tripathi, A.; Chronis, N. A doublet microlens array for imaging micron-sized objects. J. Micromech. Microeng. 2011, 21, doi:10.1088/0960-1317/21/10/105024.

19. Volkel, R.; Herzig, H.P.; Nussbaum, P.; Dandliker, R.; Hugle, W.B. Microlens array imaging system for photolithography. Opt. Eng. 1996, 35, 3323-3330.

20. Aldalali, B.; Kanhere, A.; Fernandes, J.; Huang, C.C.; Jiang, H. Fabrication of polydimethylsiloxane microlenses utilizing hydrogel shrinkage and a single molding step. Micromachines 2014, 5, 275-288.

21. Gulari, M.N.; Tripathi, A.; Chronis, N. Microfluidic-based oil-immersion lenses for high resolution microscopy. In Proceedings of the 16th International Conference on Miniaturized Systems for Chemistry and Life Sciences ( $\mu$ TAS'12), Okinawa, Japan, 28 October-1 November 2012; pp. 49-51.

22. Brückner, A.; Duparre, J.; Wippermann, F.; Leitel, R.; Dannberg, P.; Brauer, A. Ultra-compact close-up microoptical imaging system. Proc. SPIE 2010, 7786, doi:10.1117/12.860025.

23. Knieling, T.; Shaf, M.; Lang, W.; Benecke, W. Microlens array production in a microtechnological dry etch and reflow process for display applications. J. Eur. Opt. Soc. Rap. Publ. 2012, 7, doi:10.2971/jeos.2012.12007.

24. Tripathi, A.; Chokshi, T.V.; Chronis, N. A high numerical aperture, polymer-based, planar microlens array. Appl. Opt. 2009, 17, 19908-19918.

25. Pang, S.; Han, C.; Erath, J.; Rodriguez, A.; Yang, C. Wide field-of-view Talbot grid-based microscopy for multicolor fluorescence imaging. Opt. Express 2013, 21, 14555-14565.

26. Schonbrun, E.; Gorthi, S.S.; Schaak, D. Microfabricated multiple field of view imaging flow cytometry. Lab Chip 2012, 12, 268-273.

27. Cui, X.; Lee, L.; Heng, M.X.; Zhong, W.; Sternberg, P.W.; Psaltis, D.; Yang, C. Lensless high-resolution on-chip optofluidic microscopes for Caenorhabditis elegans and cell imaging. Proc. Natl. Acad. Sci. USA 2008, 105, 10670-10675.

28. Wu, J.; Zheng, G.; Lee, L.M. Optical imaging techniques in microfluidics and their applications. Lab Chip 2012, 12, 3566-3575.

29. Itatani, J.; Levesque, J.; Zeidler, D.; Niikura, H.; Pépin, H.; Kieffer, J.C.; Corkum, P.B.; Villeneuve, D.M. Tomographic imaging of molecular orbitals. Nature 2004, 432, 867-871.

30. Chen, Z.; Milner, T.E.; Dave, D.; Nelson, J.S. Optical Doppler tomographic imaging of fluid flow velocity in highly scattering media. Opt. Lett. 1997, 22, 64-66.

31. Foldscope: Microscopy for everyone. Available online: http://www.foldscope.com/ (accessed on 3 June 2014). 
32. Bogoch, I.I.; Andrews, J.R.; Speich, B.; Utzinger, J.; Ame, S.M.; Ali, S.M.; Keiser, J. Mobile phone microscopy for the diagnosis of soil-transmitted helminth infections: A proof-of-concept study. Am. J. Trop. Med. Hyg. 2013, 88, 626-629.

33. Arpa, A.; Wetzstein, G.; Lanman, D.; Raskar, R. Single lens off-chip cellphone microscopy. In Proceedings of Computer Vision and Pattern Recognition Workshops (CVPRW), 2012 IEEE Computer Society Conference on, Providence, RI, USA, 16-21 June 2012; pp. 23-28.

34. Smith, Z.J.; Chu, K.; Espenson, A.R.; Rahimzadeh, M.; Gryshuk, A.; Molinaro, M.; Dwyre, D.M.; Lane, S.; Matthews, D.; Wachsmann-Hogiu, S. Cell-phone-based platform for biomedical device development and education applications. PLoS One 2011, 6, doi:10.1371/journal.pone.0017150.

35. Frank, S.J.; Pedrotti, L.; Pedrotti, L.M.; Pedrotti, L.S. Introduction to Optics; Pearson Education: Upper Saddle River, NJ, USA, 2008.

36. Solinski, W. Olympus, Tokyo, Japan. Personal communication, 2012.

37. Basic Concepts and Formulas in Microscopy: Resolution. Available online: http://www.microscopyu. com/articles/formulas/formulasresolution.html (accessed on 22 May 2014).

38. Hubers, H.W.; Schubert, J.; Krabbe, A.; Birk, M.; Wagner, G.; Semenov, A.; Goltsman, G.; Voronov, B.; Gershenzon, E. Parylene anti-reflection coating of a quasi-optical hot-electron-bolometric mixer at terahertz frequencies. Infrared Phys. Technol. 2001, 42, 41-47.

(C) 2014 by the authors; licensee MDPI, Basel, Switzerland. This article is an open access article distributed under the terms and conditions of the Creative Commons Attribution license (http://creativecommons.org/licenses/by/3.0/). 